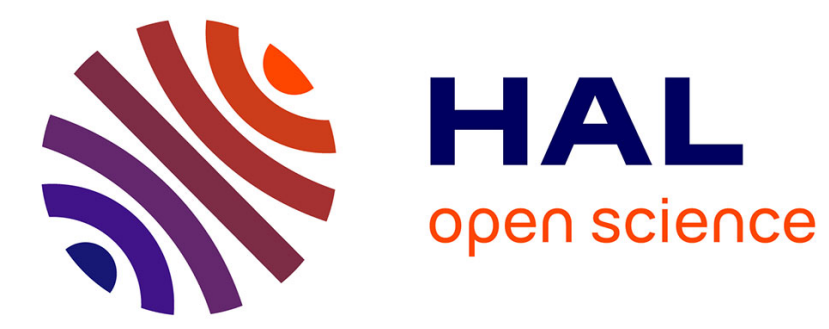

\title{
QCD studies with the hadronic final states at HERA
}

Z. Zhang

\section{To cite this version:}

Z. Zhang. QCD studies with the hadronic final states at HERA. QCD Europhysics Conference, Jul 1996, Montpellier, France. pp.91-96, 10.1016/S0920-5632(97)00021-2 . in2p3-00025511

\section{HAL Id: in2p3-00025511 https://hal.in2p3.fr/in2p3-00025511}

Submitted on 27 Jan 2006

HAL is a multi-disciplinary open access archive for the deposit and dissemination of scientific research documents, whether they are published or not. The documents may come from teaching and research institutions in France or abroad, or from public or private research centers.
L'archive ouverte pluridisciplinaire HAL, est destinée au dépôt et à la diffusion de documents scientifiques de niveau recherche, publiés ou non, émanant des établissements d'enseignement et de recherche français ou étrangers, des laboratoires publics ou privés. 


\title{
QCD studies with the hadronic final states at HERA
}

\author{
Zhiqing Zhang* \\ Laboratoire de l'Accélérateur Linéaire, \\ Université de Paris-Sud et IN2P3-CNRS, 91405 Orsay, France
}

\begin{abstract}
New and complementary results with respect to the inclusive proton structure function have been obtained in the HERA new kinematical domain by studying extensively the hadronic final states by both H1 and ZEUS collaborations. Shown here are the observation of hard partonic scattering in photoproduction and diffractive interactions, the comparison of hadron production in the central rapidity region between photoproduction and deep inelastic scattering, the precise determination of $\alpha_{s}$ over a large energy range in a single experiement, and the evidence of the hard diffractive scattering.
\end{abstract}

\section{Introduction}

With the two experiments H1 and ZEUS at HERA, a first $e p$ machine of $\sim 30 \mathrm{GeV}$ electrons (or positrons) colliding with $820 \mathrm{GeV}$ protons, new kinematical domain has been opened up compared with the previous fixed target experiments. More specifically, the centre of mass energy for photoproduction $(\gamma p)$ reactions is increased by a factor of ten up to $\sim 300 \mathrm{GeV}$, whereas values of $Q^{2}(x)$ for deep inelastic scattering (DIS) reactions are increased (decreased) by two orders of magnitude up to $\sim 10^{5} \mathrm{GeV}^{2}$ (down to $5 \cdot 10^{-5}$ at $Q^{2}=5 \mathrm{GeV}^{2}$ ). Here $Q^{2}=-q^{2}, q$ being the four-momentum of the exchanged photons and $x$ is the momentum fraction of the proton carried by the struck quark in the quark parton model. The separation between $\gamma p$ and DIS is defined in terms of the photon virtuality $\left(Q^{2}\right)$ : quasi-real photons for $\gamma p\left(Q^{2} \simeq 0 \mathrm{GeV}^{2}\right)$ and high virtual photons for DIS $\left(Q^{2}>\right.$ a few $\left.\mathrm{GeV}^{2}\right)$.

HERA also provides a unique QCD testing ground with hard interactions producing not only in DIS but also in $\gamma p$ as well as in diffractive (DIF) reactions, in which a large rapidity gap, devoid of particle production around the proton remnant direction, is observed [1].

Both $\mathrm{H} 1$ and ZEUS detectors are hermetic and multi-purpose detectors capable of measuring the complete hadronic final state particles of DIS events in addition to the scattered electron. The

\footnotetext{
*Representing the $\mathrm{H} 1$ and ZEUS collaborations. Talk given at QCD Euroconference 96, Montpellier, France, 4th - 12 th July 1996.
}

measured electron energy and angle alone fix the event kinematics and thus an inclusive cross section measurement can be straightforwardly performed. The addition of hadronic final states allows alternative methods to define the kinematics and cross-check the measurement. The latter methods reduce the radiative corrections, which can be important in certain kinematical domains when using the electron method since the dominating contribution is due to the emission of a hard initial photon collinear with the incident electron.

The determination of the cross section alone has given clues in the past decades to establish the point like constituents of the protons, to allow the observation of scaling violations, to derive parton densities and to determine the gluon distribution functions. The measurement of the hadronic system provides additional and complementary information which may not be obtained from the inclusive cross section (or structure functions).

Both H1 and ZEUS collaborations have studied extensively the hadronic final states covering a variety of subjects, which may roughly be classified into four categories in terms of means: (1) charged multiplicity, (2) charged particle spectra $p_{T}$, (3) (transverse) energy flow and (4) jet productions. Here I shall only select four of them to illustrate what can be learned at HERA by studying the hadronic final states (refer to other contributions for inclusive structure function measurement [2], diffraction [3], and heavy flavour production [4] at HERA). The emphasis is put on 
the comparison between different processes and between the HERA data and other experimental data.

\section{Charged Particle $p_{T}$ in DIF and Non- DIF $\gamma p$}

With respect to the scale $Q^{2}$ which is small for $\gamma p$, the perturbative QCD is clearly inapplicable. Fortunately, $Q^{2}$ is not the only scale governing the process. In fact, large transverse charged particles up to $8 \mathrm{GeV}$ have been observed [5] (Fig.1) indicating that the hard partonic scattering has taken place, though the dominating events are of soft nature which may be fitted with the exponential form $e^{\left(a-b \cdot \sqrt{\left.p_{T}^{2}+m_{\pi}^{2}\right)}\right.}$ as shown in the figure with the solid lines.

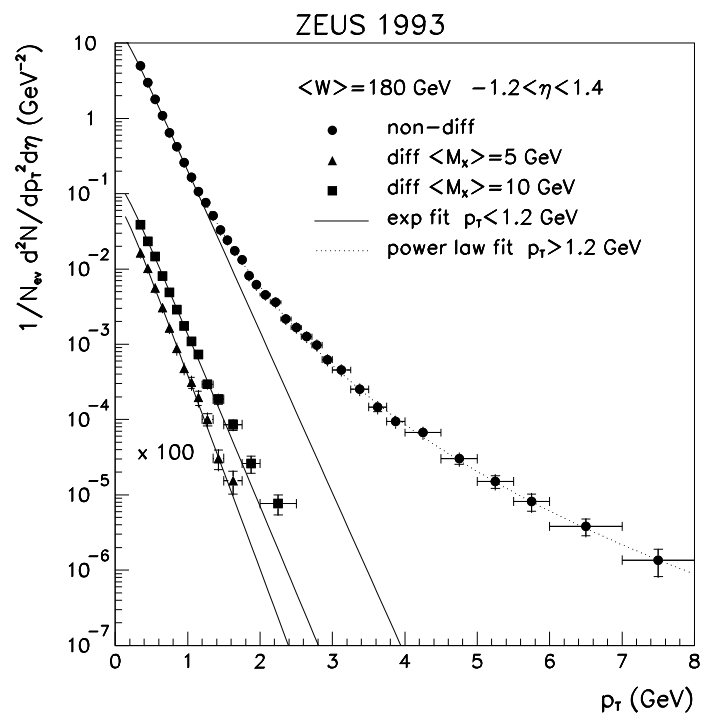

Figure 1. Inclusive transverse momentum distributions of charged particles in photoproduction events. The inner error bars indicate the statistical errors and the outer ones represent the quadratic sum of the statistical and systematic errors.

In the same figure, the $p_{T}$ spectra of DIF events at two different mass values $\left\langle M_{x}\right\rangle=5 \mathrm{GeV}$ and $\left\langle M_{x}\right\rangle=10 \mathrm{GeV}$ are compared with that of nonDIF events. Here $M_{x}$ is the invariant mass of the photon dissociation system. Again soft interactions are dominating contributions, but the slight deviation at $p_{T}>2 \mathrm{GeV}$ for $\left\langle M_{x}\right\rangle=10 \mathrm{GeV}$ indicates the hard scattering is also there in DIF $\gamma p$ reactions. Further evidence will be given in Sec.4. A comparison of the slope of the exponential fit between the DIF and non-DIF $\gamma p$ data and with previous hadron-hadron data shows i) the relevant scale for DIF is $M_{x}$ rather than the total invariant mass of the hadronic system $W$ as for non-DIF, and ii) at a same centre of mass energy the HERA data compares well with previous hadron-hadron data. Point i) is in fact more general and has been confirmed by a similar comparison between DIF and non-DIF DIS data and is the consequence of the fact that the higher the invariant mass is the larger the phase space is available for QCD radiations. Point ii) means that in soft $\gamma p$ data at low $p_{T}$ the interacting photons are hadron-like.

The high $p_{T}$ tails in Fig.1 can be understood by the following three contributions:

1. A process described by the vector meson dominance model: where the photon is pictured to fluctuate into a virtual vector meson, and the parton of the meson subsequently scatters with that of the proton.

2. Anomalous contributions: where the photon couples to a $q \bar{q}$ pair without forming a bound state, and hard scattering takes place between partons from the $q \bar{q}$ pair and from the proton.

3. Direct contribution: where the photon couples directly to the charged partons inside the proton.

The last two components are missing in $p \bar{p}$ and this explains the harder $p_{T}$ tails observed in $\gamma p$ data at HERA than in $p \bar{p}$ data.

\section{Comparison of $E_{T}$ Flow Between DIS and $\gamma p$}

Although $\gamma p$ and DIS reactions cover very different $Q^{2}$ ranges by definition, the hard scattering can take place in both processes. On the other hand, the majority of $\gamma p$ collisions show features of soft low- $p_{T}$ processes, and the DIS data at small $x$ reveal properties from soft interactions as well. Therefore it is worthwhile to compare the hadronic final state particle production in both 
event classes and to see whether it is possible to provide a universal prescription.

The transverse energy density, $1 / N \cdot d E_{T} / d \eta^{*}$, for data selected from different $Q^{2}$ in the same $y$ range ( $W \simeq 185 \mathrm{GeV})$ is shown in Fig. 2 as a function of pseudorapidity $\eta^{*}=-\ln \left(\tan \frac{\theta^{*}}{2}\right)$ in the $\gamma p$ centre of mass system (CMS) [6]. Here $\theta^{*}$ is the polar angle of a particle in the CMS frame with respect to the proton direction. A clear $Q^{2}$ dependence is seen for $\eta^{*}<-0.5$ corresponding to the photon fragmentation region, whereas there is essentially no dependence in the central rapidity region. The data are not shown at large positive rapidities, which correspond to the proton fragmentation region, due to the acceptance loss in the beam pipe.

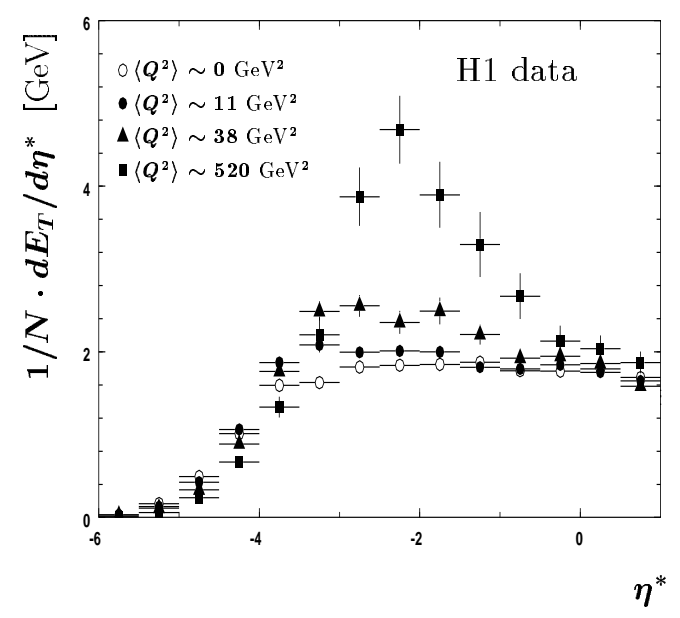

Figure 2. The flow of transverse energy $E_{T}$ in the hadronic CMS as a function of pseudorapidity $\eta^{*}$ normalized to number of events $N$.

The transverse energy density at $\eta^{*}=0$ is further shown in Fig. 3 for DIS, $\gamma p, p p$ and $p \bar{p}$ interactions as a function of $W$. It is striking that the same dependence on $W$ is observed in all interactions independent of the nature of the incoming particles: quasi-real photons, highly virtual photons, or hadrons.

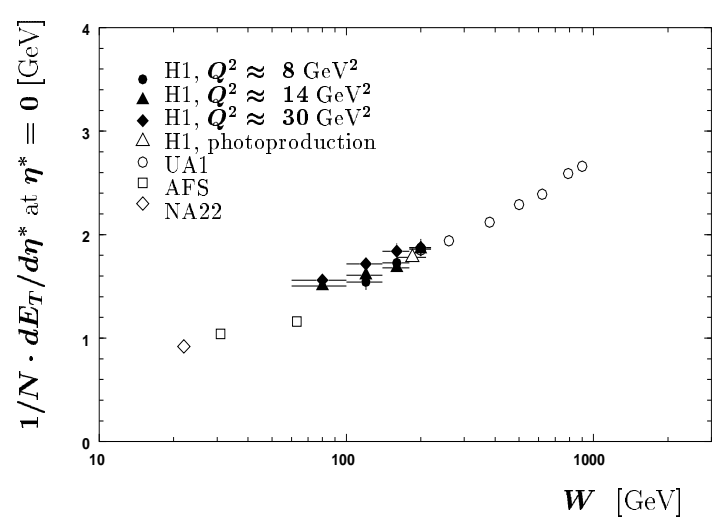

Figure 3 . The transverse energy per unit of pseudorapidity in the CMS centre region as a function of the hadronic CMS energy. Systematic pointto-point errors of $6 \%$ and an overall scale error of $9 \%$ for the $\mathrm{H} 1$ data are not shown, neither are the global scale uncertainties for other experiments.

\section{Determination of $\alpha_{s}$ from Jet Rates in DIS}

The strong coupling constant $\alpha_{s}\left(\mu^{2}\right)$ has been measured with improving precisions with many different physical observables in various experiments over a large range of centre of mass energies ranging from the $\tau$ lepton mass scale to the $Z^{0}$ mass.

The HERA experiments have the advantage that events at different energy scales (e.g. $Q^{2}$ ) are produced and thus allow the scale dependence of $\alpha_{s}$ be verified in a single experiement.

The observable is chosen to be (by identifying the factorization scale $\mu$ with $Q$ )

$$
R_{2+1}\left(Q^{2}\right)=\frac{\sigma_{2+1}\left(Q^{2}\right)}{\sigma_{\text {tot }}\left(Q^{2}\right)},
$$

following a similar method commonly used to determine $\alpha_{s}$ in $e^{+} e^{-}$annihilation. Here the subscript $2+1$ stands for two current jets plus one proton remnant jet. $\mathrm{A}(2+1)$-jet reaction arises in QCD at leading order $\left(\mathrm{LO}, \mathcal{O}\left(\alpha_{\mathcal{S}}\right)\right)$ via the emission of a hard gluon from the initial or final state quark (QCD Compton process) or via $q \bar{q}$ production in photon gluon fusion processes: $\sigma_{2+1} \propto c_{2+11} \alpha_{s}$, and at next to leading order (NLO), additional terms contribute: $\sigma_{2+1} \propto$ 
$c_{2+111_{s}} \alpha_{s}+c_{2+12} \alpha_{s}^{2}$, where the coefficients $c_{2+11(2)}$ contain the hard scattering matrix elements and the parton density functions of the incoming proton, and therefore they depend on the factorization scale $\mu$. Here the second index 1(2) stands for the order of the $\alpha_{s}$ calculation. In finite order perturbative QCD calculation, $\sigma_{2+1}$ may depend on the arbitrary renormalization scale $\mu_{R}$ due to the incomplete cancelation between $\alpha_{s}$ and the coefficients. A natural choice is $\mu=\mu_{R}=Q$ to avoid large logarithms of $Q / \mu_{(R)}$ in any finite order, making the prediction unreliable.

Experimentally, jets are reconstructed with a modified JADE cluster algorithm. The jet resolution parameter $y_{\text {cut }}$ is given by: $y_{\text {cut }}=m_{i j}^{2} / W^{2}$, where $W$ is the invariant mass of the hadronic system and $m_{i j}$ the invariant mass of any two objects. The fractional jet rates, corrected to the parton level [7], are compared in Fig.4 with two NLO calculations DISJET [8] and PROJET [9]. There is good agreement between the data and the predictions over most of the $y_{\text {cut }}$ range shown indicating that the $\alpha_{s}$ to be extracted is not sensitive to the particular value of $y_{\text {cut }}$ used. Both $\mathrm{H} 1$ and ZEUS analyses choose $y_{\text {cut }}=0.02$ because the contribution from $R_{3+1}$, which is a higher order effect, becomes negligible for $y_{\text {cut }} \geq 0.02$. Furthermore the statistics are large and the jets are resolvable at this value and the 2 -jet system

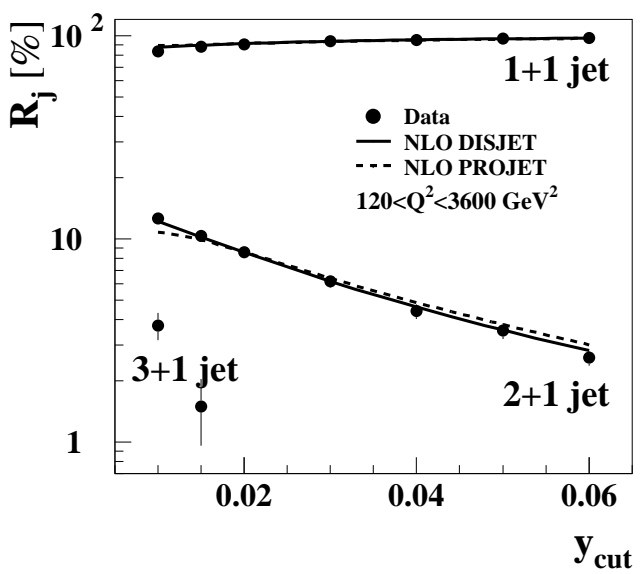

Figure 4. Jet production rate, corrected to parton level, as a function of the jet resolution parameter $y_{\text {cut }}$. has a large invariant mass. There are several other kinematical cuts, e.g. in the case of the ZEUS analysis: 1) $120<Q^{2}<3600 \mathrm{GeV}^{2}$ restricting the analysis to high $Q^{2}$, where clear jet structures are observed and hadronization uncertainties are small, 2) $0.01<x<0.1$ restricting the analysis to high $x$, where the parton densities of the proton are well known and strong correlation to the largely uncertain gluon density at low $x$ is avoided, and 3) $z=p \cdot p_{\text {jet }} / p \cdot q>0.1$ restricting the jets away from the proton remnant direction.

The results, compared with three theoretical calculations for $\Lambda_{\frac{(5)}{M S}}=100,200$, and $300 \mathrm{MeV}$, are plotted in Fig.5 as a function of $Q$ for three $Q^{2}$ ranges. The running of $\alpha_{s}$ is clearly preferred by the data. In fact, a $\chi^{2}$ fit showed that a constant $\alpha_{s}$ is ruled out at $90 \%$ confidence level.

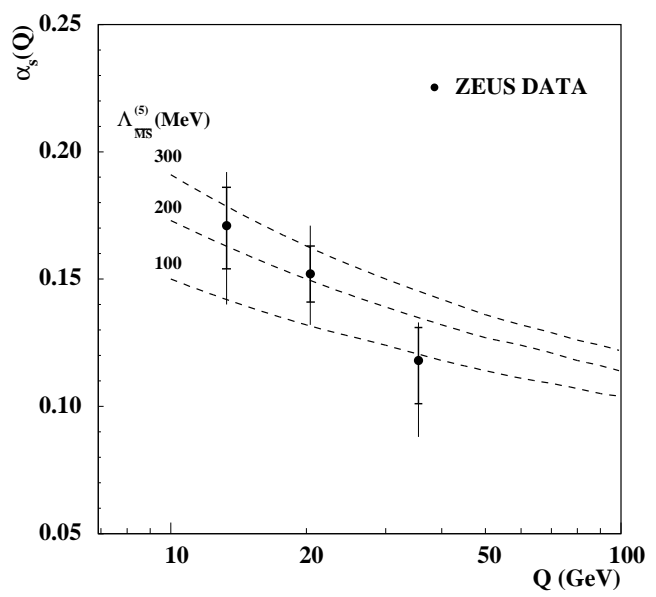

Figure 5. Measured values of $\alpha_{s}(Q)$ for three different $Q^{2}$ regions. The error bars have the same meanings as in Fig.1. The dashed curves represent $\alpha_{s}$ with $\Lambda_{\frac{(5)}{M S}}^{(5)}=100,200$, and $300 \mathrm{MeV}$.

The corresponding values of $\alpha_{s}$ at the $Z^{0}$ mass $m_{Z}$ are as follows:

$$
\begin{aligned}
\alpha_{s}\left(m_{Z}^{2}\right)= & 0.123 \pm 0.018(\text { total }) \\
\alpha_{s}\left(m_{Z}^{2}\right)= & 0.117 \pm 0.005(\text { stat })_{-0.005}^{+0.004}(\text { syst }) \pm \\
& 0.007(\text { th })
\end{aligned}
$$

from, respectively $\mathrm{H} 1$ [10] (based on 1993 data) and ZEUS [7] (based on 1994 data). The error 
(refer to original publications for more detail), which is already as competitive as other measurements, is currently dominated by theoretical uncertainty (scale uncertainty), which is expected to improve by using the two new exact NLO calculations MEPJET [11] and DISENT [12].

\section{Rapidity Gaps between Jets in $\gamma p$}

In high energy hadronic collisions, the dominant mechanism for jet production is described by a hard scattering between partons in the incoming hadrons via a quark or gluon propagator. Since this propagator carries color charge, jets are "color connected" and this leads to the production of particles throughout the rapidity region between the jets. However, if the hard scattering were mediated by the exchange of a color singlet propagator in the $t$-channel, each jet would be color connected only to the beam remnant closest in rapidity and the rapidity region between the jets would contain few final-state particles. The color singlet propagator could be an electroweak gauge boson or a strongly interacting object. However the rate, defined in terms of the gap fraction:

$$
f(\Delta \eta)=\frac{N_{\text {gap }}^{2 \text { jets }}(\Delta \eta)}{N^{2 \text { jets }}(\Delta \eta)}
$$

could be very different.

At HERA, this can be studied with the resolved photon process as shown schematically in Fig.6. This study is interesting since it may provide evidence for hard diffractive scattering between partons, complementary to studies of diffractive (hard) scattering, where the large rapidity gap appears around the proton remnant direction.

Experimentally, the jets are reconstructed from the $\gamma p$ event sample in the $W_{\gamma p}$ range $135<$ $W_{\gamma p}<280 \mathrm{GeV}$ using the cone algorithm with a cone size $R=\sqrt{\Delta \phi^{2}+\Delta \eta^{2}}=1$ and a jet threshold $E_{T}^{\mathrm{jet}}>6 \mathrm{GeV}$. The gap is defined as the absence of particles with transverse energy greater than $300 \mathrm{MeV}$. The measured gap fraction $f(\Delta \eta)$ is shown in Fig.7 [13]. It decreases exponentially as expected for processes in which color is exchanged between jets (dotted line), up to a value of $\Delta \eta \sim 3$, then reaches a constant value, which is for $\Delta \eta=3.7$ :

$$
f(\Delta \eta=3.7)=0.11 \pm 0.02(\text { stat })_{-0.02}^{+0.01}(\text { syst }) .
$$

(a)

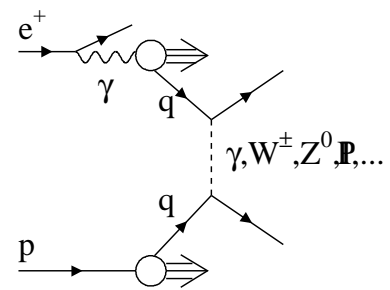

(b)

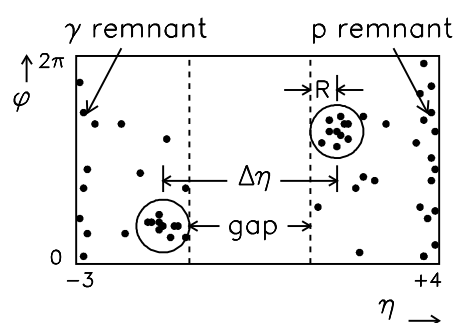

Figure 6. (a) Resolved photon process via color non-singlet exchange and (b) the rapidity gap event morphology with the black dots representing final state hadrons and the boundary illustrating the limit of the detector acceptance.

The excess above the exponential fall-off can be interpreted as evidence for hard diffractive scattering via a strongly interacting color singlet object. This object cannot be an electroweak gauge boson since the predicted rate is much too small $\left(\sigma^{\mathrm{EW}} / \sigma^{\mathrm{QCD}}<7 \cdot 10^{-4}\right)$, but is consistent with being a pomeron (a simple two-gluon model pre$\operatorname{dicts} f(\Delta \eta) \sim 0.1[14])$.

The result may be compared with the CDF and D0 results [15] of $0.0086 \pm 0.0012$ and $0.0107 \pm$ 0.0010 (stat) ${ }_{-0.0013}^{+0.0025}$ (syst). Since the gap fraction is proportional to the survival probability, which represents the probability that a secondary interaction does not occur, the higher excess may be due to the higher survival probability at lower CMS at HERA than that at Tevatron which is about $5 \%$ to $30 \%$. The different survival probabilities may also be due to the fact that one remnant jet comes from, instead of a proton at Tevatron, a photon at HERA, where the average fraction of the photon energy participating in the production of the two jets is rather high and thus the fraction of the photon energy available for secondary interaction is low. 


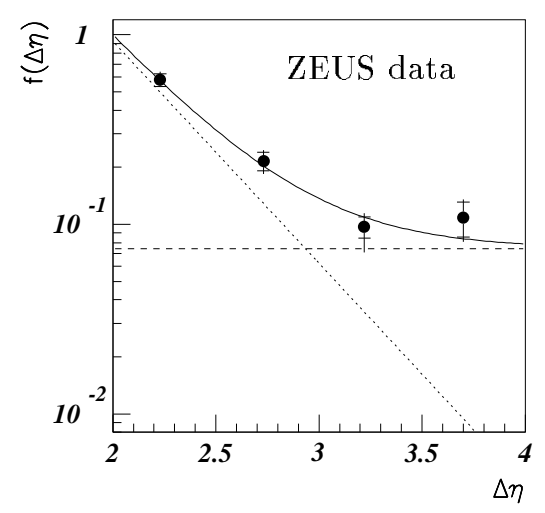

Figure 7. Measured gap-fraction $f(\Delta \eta)$. The error bars have the same meanings as in Fig.1. The expected behaviour for the non-singlet contribution is shown as the dotted line and the color singlet contribution as the dashed line.

\section{Summary}

Based on the data taken in 1992-1994 by both H1 and ZEUS collaborations, corresponding to an increasingly integrated luminosity of up to $5 \mathrm{pb}^{-1}$ per year, much has been learned in the new HERA kinematical domain by studying the hadronic final states. These include among what have been shown:

- Hard partonic scattering is present not only in DIS, but also in $\gamma p$ and diffractive interactions, allowing the perturbative QCD be tested in different processes.

- The hadron production is found to be rather universal in the central rapidity region in the $\gamma p$ CMS independent of the nature of the incoming particles, showing the potential of revealing a unique description for $\gamma p$ and low $x$ DIS reactions.

- The fractional jet rates in DIS have provided a new precise measurement of $\alpha_{s}$ and the large scale variation of $Q^{2}$ has allowed the running of $\alpha_{s}$ be tested in a single experiment.

- Both diffractive (hard) scattering and hard diffractive scattering have been observed at HERA. The unusual feature of these events is consistent with the exchange of a pomeron-like color singlet object.

With expected large increase of integrated luminosity and the detector updates (e.g. the installation of the proton and neutron spectrometers in the outgoing proton direction), a lot of exciting results will become available, which may turn out to be essential for further testing QCD and deepening our understanding of QCD dynamics in the low $x$ regime.

\section{Acknowledgements}

It is a pleasure to thank all the members of the $\mathrm{H} 1$ and ZEUS collaborations, whose work allow me to present these results. I would like to thank $\mathrm{S}$. Narison for organizing this pleasant conference.

\section{REFERENCES}

1. ZEUS Collab., M. Derrick et al., Phys. Lett. B315 (1993) 481; H1 Collab., T. Ahmed et al., Nucl. Phys. B429 (1994) 477.

2. F. Chlebana, these Proceedings.

3. C. Niebuhr, these Proceedings.

4. U. Karshon, these Proceedings.

5. H1 Collab., I. Abt et al., Phys. Lett. B328 (1994) 241; ZEUS Collab., M. Derrick et al., Z. Phys. C67 (1995) 227.

6. H1 Collab., S. Aid et al., Phys. Lett. B358 (1995) 412.

7. ZEUS Collab., M. Derrick et al., Phys. Lett. B363 (1995) 201.

8. T. Brodkorb and E. Mirkes, Univ. of Wisconsin, MADPH-94-821 (1994).

9. D. Graudenz, Comp. Phys. Comm. 92 (1995) 65.

10. H1 Collab., T. Ahmed et al., Phys. Lett. B346 (1995) 415.

11. E. Mirkes and D. Zeppenfeld, MADPH-96935 (1996).

12. S. Catani and M. Seymour, CERN-TH/9628,29 (1996).

13. ZEUS Collab., M. Derrick et al., Phys. Lett. B369 (1996) 1.

14. J. D. Bjorken, Phys. Rev. D47 (1992) 101.

15. CDF Collab. F. Abe et al., Phys. Rev. Lett. 74 (1995) 855; D0 Collab., S. Abachi et al., Phys. Rev. Lett. 72 (1994) 2332, and A. Goussiou, these Proceedings. 